\title{
A Deformable Vessel Model with Single Point Initialization for Segmentation, Quantification and Visualization of Blood Vessels in 3D MRA
}

\author{
Marcela Hernández-Hoyos, Alfred Anwander, Maciej Orkisz, \\ Jean-Pierre Roux, Philippe Douek, Isabelle E. Magnin \\ CREATIS, CNRS Research Unit (UMR 5515) affiliated to INSERM, Lyon, France \\ CREATIS, INSA 502, 20 av. Albert Einstein, 69621 Villeurbanne cedex, France \\ marcela.hernandez@creatis.insa-lyon.fr \\ http://www.creatis.insa-lyon.fr
}

\begin{abstract}
We deal with image segmentation applied to three-dimensional (3D) analysis of of vascular morphology in magnetic resonance angiography (MRA) images. The main goal of our work is to develop a fast and reliable method for stenosis quantification. The first step towards this purpose is the extraction of the vessel axis by an expansible skeleton method. Vessel boundaries are then detected in the planes locally orthogonal to the centerline using an improved active contour. Finally, area measurements based on the resulting contours allow the calculation of stenosis parameters. The expansible nature of the skeleton associated with a single point initialization of the active contour allows overcoming some limitations of traditional deformable models. As a result, the algorithm performs well even for severe stenosis and significant vessel curvatures. Experimental results are presented in 3D phantom images as well as in real images of patients.
\end{abstract}

\section{Introduction}

Atherosclerosis is the principal acquired affection of the vascular wall. It remains the number one cause of death among people older than 60 years. Its major complication is the arterial stenosis. Precise stenosis quantification is necessary for diagnosis purposes and treatment planning.

Accurate quantitative measurements of vessel morphology require previous vessel segmentation. Two main approaches for vessel segmentation can be distinguished. The first one relies on purely photometric criteria, mainly on thresholding and regiongrowing techniques [1]-[4]. Its major advantage is its generally simple implementation. However, a further modelling step is necessary to extract meaningful measurements from thus segmented images. The second approach exploits the geometrical specificity of the vessels, in particular the notions of orientation and tubular shape. Most of these approaches consists in vessel-tracking [5]-[9] and use (often implicitly) a generalized-cylinder model, i.e. an association of an axis (centerline) and a surface (vessel wall). Consequently, the segmentation process involves two tasks: centerline extraction and vessel contour detection (most often using a $2 \mathrm{D}$ deformable model) in the planes usually perpendicular to the axis. This S.L. Delp, A.M. DiGioia, and B. Jaramaz (Eds.): MICCAI 2000, LNCS 1935, pp. 735-745, 2000.

(c) Springer-Verlag Berlin Heidelberg 2000 
procedure results in a stack of $2 \mathrm{D}$ contours along the vessel, allowing quantitative cross-section measurements and visualization by means of triangulation-based rendering. Other more recent approaches use 3D deformable models of surface [10][11]. Although deformable models have been shown to be useful for segmenting images from various imaging modalities most of them, for good convergence, require an initialization close to the final solution.

In this context, we propose a novel implementation for vessel segmentation using deformable models for vessel centerline and contours. The principal contribution of our approach compared to the others resides in the simplicity of the model initialization and in the flexibility of its evolution. The algorithm starts from a single point inside the vascular segment to extract, and a simulated propagation of this point along the vessel defines its skeleton. In a similar way, the active model for the contour detection starts from the single point determined by the axis position in the orthogonal plane.

\section{Method}

On the basis of the discrete generalized-cylinder model [12], we propose a segmentation technique divided into two steps. First, the extraction of the vessel central axis is performed using an expansible vessel skeleton. Contours are then detected in the planes locally orthogonal to the centerline with an improved 2D active contour.

\subsection{Expansible Vessel Skeleton}

The vessel axis extraction is achieved by an expansible skeleton method. It is based on a tracking strategy, which starts from a user-selected point within the vessel, and then iteratively estimates the subsequent axis points. Geometrically, the axis is defined by a list of points embedded in the image volume $(\mathrm{x}, \mathrm{y}, \mathrm{z}) \in \mathfrak{R}^{3}$. We consider the expansion of the axis as an iterative process where at each iteration, a new point is added to the model. At iteration $k$, the axis is represented by:

$$
A_{k}=\left\{\mathbf{p}_{i}, i=1 . . k\right\}
$$

where $\mathbf{p}_{i}$ is the position of point $i$ and $k$ is the total number of points at this iteration. Point generation is a two-step procedure. First, a prediction of the new point position is obtained, based on the vessel local orientation at the last axis point. This position is then corrected under the influence of image forces and shape constraints.

Prediction. Let $\mathbf{p}_{i}$ be the point added to the axis during the last iteration, the position of the next point is predicted by applying a "constant velocity" displacement of $\mathbf{p}_{i}$ along the vessel local orientation. The vessel local orientation is estimated by inertia moment minimization for a small volume (box or cell) centered on the current point $\mathbf{p}_{i}$. Let us recall that, for an axis $\chi$ passing through the gravity center, the inertia moment of the box can be expressed as: 


$$
M_{\chi}=\mathbf{e}_{\chi}^{T} \mathbf{M} \mathbf{e}_{\chi}
$$

$\mathbf{e}_{\chi}$ is a unit vector corresponding to $\chi, \mathbf{M}$ is the inertia matrix defined with respect to any coordinate system whose origin is the gravity center. The vessel orientation corresponds to the axis $\chi$ for which $M_{\chi}$ is minimum. It is given by the eigenvector $\mathbf{e}_{\chi \min }$ associated with the smallest eigenvalue of the inertia matrix $\mathbf{M}$. The expression of the new predicted point $\hat{\mathbf{p}}_{i+1}$ is:

$$
\hat{\mathbf{p}}_{i+1}=\mathbf{p}_{i}+\mu s_{i} \mathbf{e}_{\chi \min } .
$$

Since $\mathbf{e}_{x \min }$ is only an orientation unit vector, $s_{i}$ and $\mu$ are included in order to control respectively the direction and the magnitude of the movement. The latter is a fixed parameter, while $s_{i}$ is computed using the displacement vector $\mathbf{d}_{i}$ from the previous iteration:

$$
s_{i}=\operatorname{sign}\left(<\mathbf{e}_{\chi \min }, \mathbf{d}_{i}>/\left\|\mathbf{e}_{\chi \min }\right\| \cdot\left\|\mathbf{d}_{i}\right\|\right) \text {, where } \mathbf{d}_{i}=\mathbf{p}_{i}-\mathbf{p}_{i-1} .
$$

Correction. The inertia-based approach described above provides a convenient way to automate the axis extraction of most regular vascular structures. Moreover it offers a good noise-robustness and gives satisfactory results even for small vessels [13]. It has been shown however, that the algorithm constructs an absurd axis for some structures such as the one presented in Fig. 1 [7].

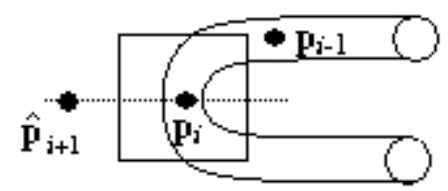

Fig. 1. Erroneous computation of the new point based on the main axis of inertia alone (the rectangle represents the cell used for the inertia moments computation)

We thus propose to estimate the position of the new point by adding a correction to the predicted position $\hat{\mathbf{p}}_{i+1}$. This correction results from submitting the point $\hat{\mathbf{p}}_{i+1}$ to two kinds of elementary forces: external and internal ones. The external forces are used to attract the point toward a position which has a high likelihood of lying along the vessel centerline. Assuming that the maximum of the MR signal is reached at the vessel axis, we propose two external forces, which respectively attract the point towards a local maximum of intensity and towards the gravity center of a box centered in $\hat{\mathbf{p}}_{i+1}$. The total displacement due to these forces can be written as:

$$
\boldsymbol{\Delta}_{i+1}^{\mathrm{ext}}=\boldsymbol{\Delta}_{i+1}^{\mathrm{gc}}+\boldsymbol{\Delta}_{i+1}^{\max }, \text { where: }
$$


$-\boldsymbol{\Delta}_{i+1}^{\mathrm{gc}}=-\eta\left(\mathbf{p}_{i+1}^{\mathrm{gc}}-\hat{\mathbf{p}}_{i+1}\right)$ is the displacement due to the attraction force exerted by the local gravity center $\mathbf{p}_{i+1}^{\mathrm{gc}}$, weighted by the coefficient $\eta$.

$-\boldsymbol{\Delta}_{i+1}^{\max }=-\kappa\left(\mathbf{p}_{i+1}^{\max }-\hat{\mathbf{p}}_{i+1}\right)$ is the displacement due to the attraction force exerted by the local maximum intensity point $\mathbf{p}_{i+1}^{\max }$, controlled by $\kappa$.

The internal forces are used to impose smoothness and continuity constraints, in order to limit oscillations and reduce the noise-sensitivity. These forces provide the internal constraints over the first and second order discrete derivatives and act like the regularization terms used in the classical snakes internal energy [14]. The expression of the total displacement due to the internal forces is:

$$
\boldsymbol{\Delta}_{i+1}^{\mathrm{int}}=-\alpha_{a}\left(\hat{\mathbf{p}}_{i+1}-\mathbf{p}_{i}\right)-\beta_{a}\left(\mathbf{p}_{i-1}-\mathbf{2} \mathbf{p}_{i}+\hat{\mathbf{p}}_{i+1}\right) .
$$

Two coefficients dictate the physical characteristics of the axis: $\alpha_{a}$ controls its elasticity while $\beta_{a}$ controls its flexibility. Taking into account the displacements (5) and (6), the estimated new point position is:

$$
\mathbf{p}_{i+1}=\hat{\mathbf{p}}_{i+1}+\Delta_{i+1}^{\mathrm{ext}}+\Delta_{i+1}^{\mathrm{int}} .
$$

When the axis has been tracked, we proceed to the vessel lumen boundary extraction using 2D active contours applied orthogonally to the centerline. For this purpose, the centerline is interpolated using a B-spline curve and the image volume is re-sliced.

\subsection{Vessel Contour Extraction}

The active contour (snake) model is used to ensure continuity and smoothness of the detected boundaries. A compromise is sought between these internal constraints and external forces attracting the snake to image features. This concept was introduced by [14], but convergence of this initial model was strongly dependent on its initialization. Much work was then devoted to overcome this problem. We first recall basic concepts concerning the active contours, then we propose a new implementation, which only needs a single point as initialization. The contour is automatically initialized by the cross-section between the vessel axis and the orthogonal image plane.

Active Contour Model. A deformable contour model is defined by a parametric curve $\mathbf{v}(s, t)=(x(s, t), y(s, t))^{T}$, which evolves in time $t$ and space, and its energy $E(\mathbf{v})=E_{\text {int }}(\mathbf{v})+E_{\text {ext }}(\mathbf{v})$, where $s \in[0,1]$ is the arc length. The energy $E_{\text {int }}(\mathrm{v})$, corresponding to internal forces, imposes constraints on the first and second derivatives of the curve: 


$$
E_{\text {int }}(\mathbf{v})=E_{\text {elast }}(\mathbf{v})+E_{\text {flex }}(\mathbf{v})=\alpha_{c} \int_{0}^{1}\left|\frac{\partial \mathbf{v}(s, t)}{\partial s}\right|^{2} d s+\beta_{c} \int_{0}^{1}\left|\frac{\partial^{2} \mathbf{v}(s, t)}{\partial s^{2}}\right|^{2} d s
$$

and the coefficients $\alpha_{c}$ and $\beta_{c}$ play the same role as $\alpha_{a}$ and $\beta_{a}$ in (6). There are two kinds of external forces represented by a potential energy $E_{\text {ext }}(\mathbf{v})=\int_{s=0}^{1} P(\mathbf{v}(s, t)) d s$ : image forces and a balloon force. The link between potentials $P_{i}(\mathbf{v})$ and forces is given by : $F_{i}(\mathbf{v})=-\nabla P_{i}(\mathbf{v})$. The image forces are usually designed to attract the snake toward strong intensity gradients. One can also use prior knowledge about the vessel outline as function of the intensity, so that the potential energy function has a minimum at the vessel boundary. With an initialization far from any edge, the active contour would collapse. One solution consists in adding an external balloon force $F_{b}$ [15], which acts like an inflating pressure, and makes the active contour model move without any image gradient. It is expressed as $F_{b}(\mathbf{v})=b \mathbf{n}(s, t)$, where $\mathbf{n}(s, t)$ is the normal unitary vector oriented outward at the point $\mathbf{v}(s, t)$, and $b$ is a coefficient which controls the inflation. The total external force $F_{\text {ext }}(\mathbf{v})$ is a weighted sum of these forces.

The parametric curve $\mathbf{v}(s)$ is fitted to the image by minimization of the energy $E(\mathrm{v})$. The minimization is a temporal and spatial discrete process, using a finite number $N$ of snake points $\mathbf{v}(i, k)$ that approximate the snake as a polygon. The partial derivatives in (8) are approximated by finite differences $\partial \mathbf{v}(s, t) / \partial s \approx(\mathbf{v}((i+1), k)-\mathbf{v}((i-1), k)) / 2 h$, with $h$ the discretization step of the snake points on the contour. The snake is iteratively deformed, explicitly using the external forces $F_{\text {ext }}(\mathbf{v})$ at each point of the snake, while implicitly minimizing the internal energy. The new position $\mathbf{v}(i, k)$ at time step $t$ is computed by solving the associated Euler equations. These equations can be solved by matrix inversion:

$$
\mathbf{v}(i, k)=[\gamma \mathbf{I}+\mathbf{A}]^{-1} \cdot\left[\gamma \cdot \mathbf{v}(i,(k-1))+F_{\text {ext }}(\mathbf{v}(i,(k-1)))\right] .
$$

This equation is referred to as the evolution equation of the snake [14]. The matrix A represents a discretized formulation of the internal energy. The damping parameter $\gamma$ controls the snake deformation magnitude at each iteration.

Proposed Numerical Implementation and Parameterization. The discretization step $h$ depend on the number of snake points $N$ and, initially, is equal to the distance between the snake points. The evolution equation (9) is only valid if the snake points are equally distanced and the step $h$ is unchanged. After each iteration however, the length of a snake grows due to external force, and $h$ does not correspond to the real distance between the snake points. The internal energy, especially the term $E_{\text {elast }}(\mathbf{v})$ 
associated with the contour's tension, grows with the snake length and stops the snake before the boundary is reached. This is particularly annoying if the initialization is far from the final position. In this case, the model needs to be resampled with a new (higher) number of snake points $N$, or/and a new step $h$. The $N$ by $N$ matrix $[\gamma \mathbf{I}+\mathbf{A}]$, as well as its inverse have to be recomputed. This is a time-consuming task which limits the classical snake model application in many cases.

To make the snake scale independent and allow an initialization by a single pixel in the image, we propose a new numerical scheme for the snake energy minimization. It was designed to preserve the validity of the equation (9) in spite of the evolution of the snake's size. It only needs a single computation of the matrices $[\gamma \mathbf{I}+\mathbf{A}]$ and $[\gamma \mathbf{I}+\mathbf{A}]^{-1}$, and uses a fixed number of points for all the iterations. We propose to scale the snake size at each iteration, in order to normalize the internal energy of the model with a fixed discretization step $h^{\prime}=1$. The scaled snake $\mathbf{v}^{\prime}(i,(k-1))$ has a normalized length equal to the number of its points. It is obtained from the actual snake using the average distance $\bar{h}_{k-1}$ between its points:

$$
\mathbf{v}^{\prime}(i,(k-1))=\mathbf{v}(i,(k-1)) / \bar{h}_{k-1} .
$$

The scaled snake is deformed according to the evolution equation (9), using the external forces $F_{\text {ext }}$ from the non-scaled snake. Hence, the new (scaled) positions for the snake points $\mathbf{v}^{\prime}(i, k)$ are:

$$
\mathbf{v}^{\prime}(i, k)=[\gamma \mathbf{I}+A]^{-1} \cdot\left[\gamma \cdot \mathbf{v}^{\prime}(i,(k-1))+F_{e x t}(\mathbf{v}(i,(k-1)))\right] \text {. }
$$

Let $\Delta \mathbf{v}^{\prime}(i, k)$ be the snake deformation vectors for each snake point:

$$
\Delta \mathbf{v}^{\prime}(i, k)=\mathbf{v}^{\prime}(i, k)-\mathbf{v}^{\prime}(i,(k-1)) \text {. }
$$

The deformation vectors are computed with a normalized internal energy, and the deformation is directly applied on the non-scaled snake:

$$
\mathbf{v}(i, k)=\mathbf{v}(i,(k-1))+\Delta \mathbf{v}^{\prime}(i, k) .
$$

In this new position, the snake points are redistributed along the snake polygonal outline, to ensure an equal distance $h_{k}$ between all snake points for the next iteration.

\section{Results and Applications}

\subsection{Stenosis Quantification}

In order to assess the precision of the methods described above, we tested them on phantom images. Our Computer Assisted Design (CAD) phantoms are cylinders whose internal surface represents the endoluminal shape of the vessel (Fig. 3). Their internal reference diameter is $6 \mathrm{~mm}$ and each of them comprises 2 stenosis. We tackled the problem of area measurements for stenosis quantification. Cross-sectional 
areas from the normal ("healthy") parts of the vessel were used to calculate the average normal area $\overline{S_{n}}$ and the corresponding coefficients of variation defined as:

$$
C V_{\sigma}=\frac{\sigma_{s}}{\overline{S_{n}}} \times 100 \quad C V_{\max }=\frac{\max \left(S_{\max }-\overline{S_{n}}, \overline{S_{n}}-S_{\min }\right)}{\overline{S_{n}}} \times 100
$$

where $\sigma_{s}$ is the standard deviation of the measured area, $S_{\max }$ and $S_{\min }$ are respectively the maximum and minimum normal area. The degree of stenosis was computed as the pathological cross-sectional area $S_{m}$ and the $\overline{S_{n}}$ ratio, as indicated in Fig. 2.

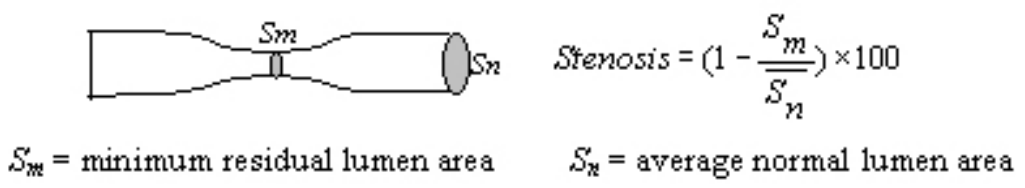

Fig. 2. Stenosis quantification

The stenosis severity estimation was quantitatively evaluated by comparing it to the true theoretical stenosis values of the phantoms. A summary of quantitative results for these data is given in Fig. 3. The CV coefficients $\left(C V_{\sigma}<2 \%, C V_{\max }<5 \%\right)$ indicate a good stability of the vessel contour extraction results. The fluctuations can be attributed to the partial volume effect. Indeed, let us consider the case of the phantom (a) which presents the greatest CV. The cross-sectional area from a normal part (diameter $=6 \mathrm{~mm}$ ) is $28.27 \mathrm{~mm}$. Its voxel size is $0.78 \mathrm{~mm} \times 0.78 \mathrm{~mm} \times 0.76 \mathrm{~mm}$. It means that each pixel missing (or exceeding) in the extracted surface, represents an error of $2,15 \%$. This shows that our results contain not more than one or two misclassified pixels, which is a correct value if one takes into account the partial volume effect. Moreover, the stenosis quantification absolute errors are not more significant than the above mentioned variations and could be explained by the same reasons. The slightly larger errors for the phantom (b) may be due to a more important partial volume effect, since the image, in this case, was acquired with a coarser resolution.

\subsection{Qualitative Results}

The axis position as well as the cross-sectional contour shape have been qualitatively evaluated on data from 7 patients with stenosis located in different arteries: carotid, renal and lower limbs. According to a visual appreciation, the results were satisfactory for large vessels as well as for small and low intensity ones. Fig. 4 shows examples of successful centerline extractions for vessels of different sizes in patient images. 
(a)

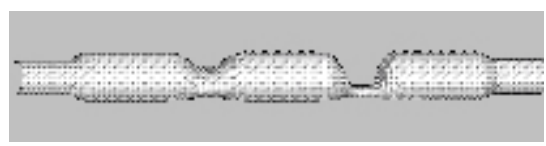

(b)

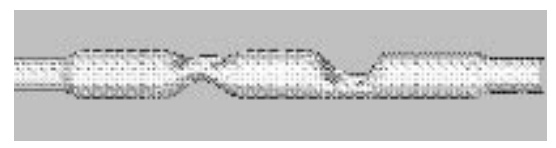

CAD images of the phantom

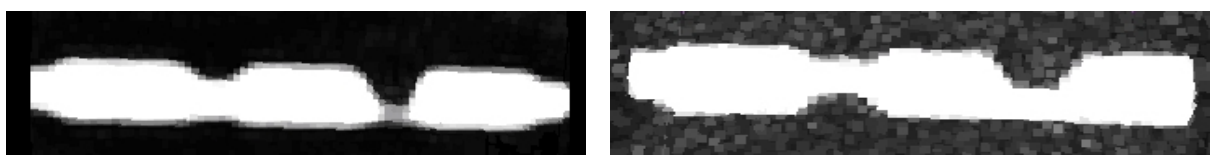

Maximum Intensity Projection (MIP)
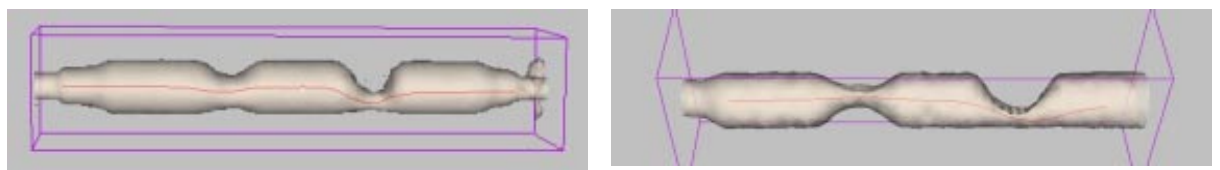

Surface rendering of the segmented MR image with the extracted centerline

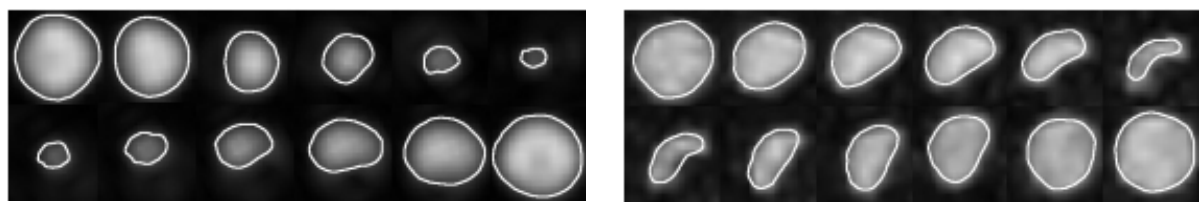

A few planes orthogonal to the extracted centerline.

The extracted contours are superimposed onto the images
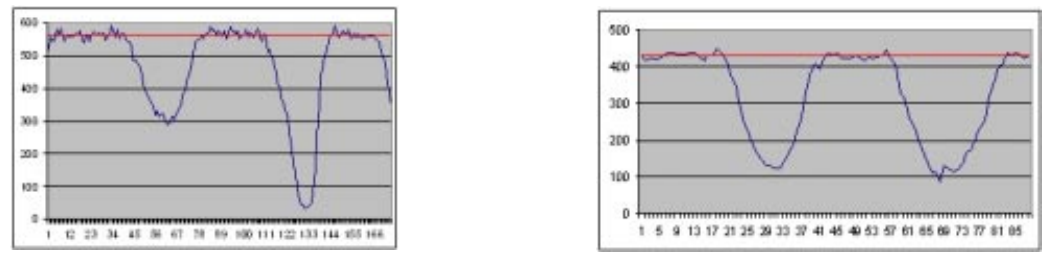

Cross-sectional area. The average normal cross-sectional area is indicated by the red line

\begin{tabular}{|l|l|}
\hline Stenosis & Estimated \\
\hline $50 \%$ & $48.33 \%$ \\
\hline $95 \%$ & $94.33 \%$ \\
\hline
\end{tabular}

\begin{tabular}{|l|l|}
\hline Stenosis & Estimated \\
\hline $75 \%$ concentric & $70.53 \%$ \\
\hline $75 \%$ eccentric & $73.80 \%$ \\
\hline
\end{tabular}

$$
\begin{aligned}
& C V_{\sigma}=1.92 \% \\
& C V_{\max }=4.91 \%
\end{aligned}
$$

$$
\begin{aligned}
& C V_{\sigma}=1.98 \% \\
& C V_{\max }=4.29 \%
\end{aligned}
$$

Fig. 3. Results from phantom data. Column (a): Phantom with a 50\% and a $95 \%$ stenosis, both elliptic and eccentric. Column (b): Phantom with a 75\% semi-lunar, eccentric stenosis and a $75 \%$ semi-lunar concentric stenosis. 


\subsection{Visualization Application}

Virtual endoscopic or fly-through methods, combining the features of endoscopic viewing with cross-sectional and volumetric imaging, are of great interest for the physicians. At present, some of clinical workstations provide this functionality, but their major drawback is that the user needs to define the path manually. This manual planning of flight paths is a tedious, imprecise and time-consuming task. A direct application of our vessel axis extraction method in the field of visualization is the automation of the path construction for guiding virtual endoscopic exploration of vessels. Fig. 5 shows an example of aorta exploration: the vessel axis is visualized at the same time as the vascular walls. In this case, the translucent wall allows the operator to see the collateral small vessels like the renal arteries, which may be used as reference points for trajectory location.

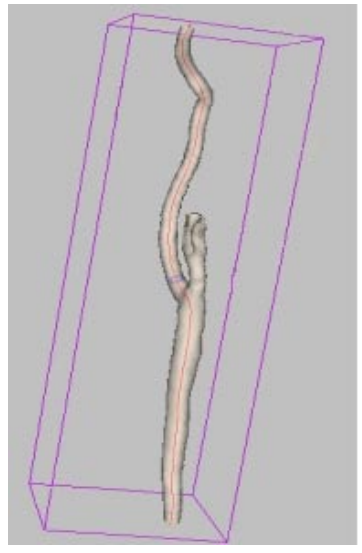

(a)

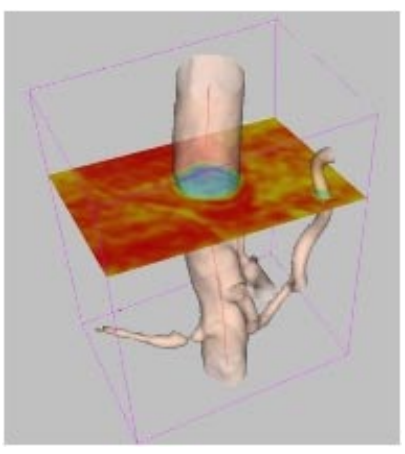

(b)

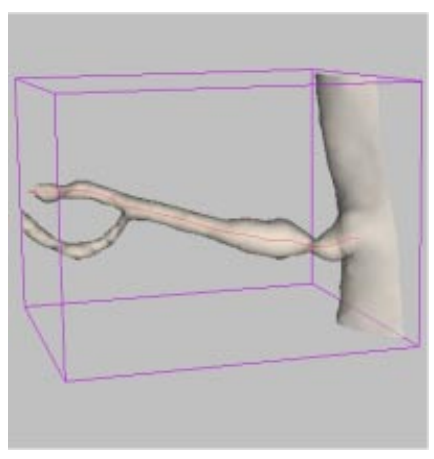

(c)

Fig. 4. Vessel axis extraction results. (a) Carotid artery and its axis. (b) Aorta central axis and orthogonal plane (c) Severely stenosed renal artery and its extracted axis
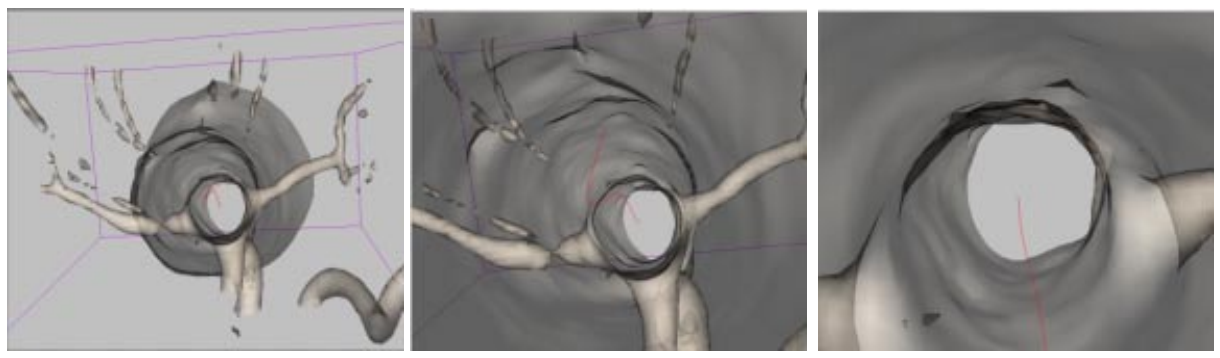

Fig. 5. Aorta virtual endoscopy guided by the detected centerline 


\section{Discussion and Conclusion}

We devised a vessel segmentation method applied to stenosis quantification in 3D MRA images. The method was qualitatively and quantitatively evaluated. The visual appraisal of the results shows a precise estimation of the vessel axis position and orientation as well as of contour location. Quantitative results show that the stenosis severity was estimated with good accuracy even for severe stenosis.

Concerning the vessel axis extraction, our method differs from other vascular network extraction techniques founded on second derivatives [7][16] or on curvatures [17], proved to be noise-sensitive. Hence our first objective was to reduce the noisesensitivity in order to cope even with very small and low contrast vessels. Due to its expansible nature, the centerline extraction algorithm overcomes the initialization limitations of deformable models as well as some of geometrical flexibility constraints such as the adaptation to significant curvatures. Nevertheless, we remarked that the results are dependent on the size of the cell used for the inertia moments computation and it needed to be adjusted according to the size to the vessel to be extracted. A multiscale approach could be considered to cope with vessel width variability.

Concerning the vessel contour detection, its advantages can be summarized as follows. The contour motion is independent of the scale of the snake. With the damping parameter $\gamma$ (eq. 11), the snake can move with a controlled speed of about one pixel/ iteration throughout its evolution, and thus avoids missing energy minima on its way. Furthermore, the method does not need much computation time compared to the classical snake model: the scaling of the control points is a simple multiplication for each point and the points redistribution is a fast operation. The normalized snake with a fixed number of control points does not need to change the matrix of the snake model, and is initialized only once. This makes the snake much faster. The deformation is shape independent, and the final outline is independent from the initialization. This allows an initialization with a single pixel, and growing in each direction while keeping a smooth outline.

Nevertheless, the proposed approach shows some limitations. Based on a generalized-cylinder model, it cannot deal with bifurcations. At present, the algorithm succeeds in extracting the vessel axis even at the joint points, but it tracks only one branch at a time. Additional work is necessary to process the whole vessel tree. Furthermore, reliable boundary detection should not be limited to 2D contours in the planes locally orthogonal to the axis, but should be carried out in a 3D neighborhood.

\section{Acknowledgements}

This work is in the scope of the scientific topics of the GDR-PRC ISIS research group of the French National Center for Scientific Research (CNRS). 


\section{References}

1. X. Hu, N. Alperin, D. Levin, K. Tan, M. Mengeot - Visualization of MR angiographic data with segmentation and volume-rendering techniques, J Magnetic Resonance Imaging 1, 539-546, 1991

2. D.L. Wilson, J.A. Noble - Segmentation of cerebral vessels and aneurysms from MR angiography data, Information Processing in Med Imaging. $15^{\text {th }}$ Int. Conf, Springer, Berlin, 423-428, 1997

3. Y. Masutani, T. Schiemann, K-H. Höne, Vascular shape segmentation and structure extraction using a shape-based region-growing model, MICCAI'98, Boston USA, 12421249, 1998

4. A.C.S. Chung, J.A. Noble, Statistical 3D vessel segmentation using a Rician distribution, MICCAI'99, Cambridge UK, 82-89, 1999

5. B. Verdonck, I. Bloch, H. Maître - Accurate segmentation of blood vessels from 3D medical images, ICIP'96, Lausanne , 311-314, 1996

6. B. Nazarian, C. Chédot, J. Sequeira, Automatic reconstruction of irregular tubular structures using generalized cylinders, Revue de CFAO et d'informatique graphique, 11(1-2), 11-20, 1996

7. C. Lorenz, I-C. Carlsen, T.M. Buzug, C. Fassnacht, J. Weese - Multi-scale line segmentation with automatic estimation of width, contrast and tangential direction in 2D and 3D medical images, CVRMed/MRCAS'97, Grenoble, France, 233-242, 1997

8. O. Wink, W.J. Niessen M.A. Viergever, Fast quantification of abdominal aorta aneurysmes from CTA volumes, MICCAI'98, Boston USA, 138-145, 1998

9. KC. Wang, R.W. Dutton, C.A. Taylor, Improving geometric model construction for blood flow modeling, IEEE Engineering in Medicine and Biology, 18(6), 33-39, 1999

10. A.F. Frangi, W.J. Niessen et al, Model-Based quantitation of 3-D magnetic resonance angiographic images, IEEE Transactions on Medical Imaging, 18(10), 946-956, 1999

11. A. Bulpitt, E. Berry, An automatic 3D deformable model for segmentation of branching structures compared with interactive region growing, Med Image Understanding Anal, Leeds UK, 25-28, 1998

12. U. Shani, D. Ballard, Splines as embeddings fot generalized cylinders, CVGIP'84, 27(2), 1984.

13. M. Hernández-Hoyos, M. Orkisz et al, Inertia-based vessel axis extraction and stenosis quantification in 3D MRA images, CARS'99, Paris, 189-193,1999

14. M. Kass, A. Witkin, D. Terzopoulos, Active contour models, Int. J Computer Vision, 1, 321-331, 1988

15. L. D. Cohen, On Active Contour Models and Balloons, Computer Vision, Graphics and Image Processing: Image Understanding, 53(2), 211-218, 1991

16. Y. Sato, S. Nakajima, et al. - 3D Multi-scale line filter for segmentation and visualization of curvilinear structures in medical images, CVRMed/MRCAS'97, Grenoble, France, 213-222, 1997

17. V. Prinet, O. Monga - Vessels representation in 2D and 3D angiograms, CARS'97, 240245, 1997 